\title{
Herausforderung: Maritime Sicherheit
}

\author{
Rainer Brinkmann*/Dirk Peters**
}

\begin{abstract}
Maritime Security is gaining significance in the field of security policy for various reasons. Attending to maritime security is a core national interest of the Federal Republic of Germany. But maritime security cannot be achieved by the effort of a single nation. It is global in scope. In consequence, numerous international initiatives are pursuing the aim of sharpening public awareness of maritime security issues, tightening the surveillance of sea areas and extending cooperation in detecting and defending against dangers while respecting national differences. By creating the Maritime Security Centre, Germany has established the preconditions for intensifying the cooperation of state and federal enforcement agencies while involving the German Navy. In particular the involvement of the Navy, which could make a substantial contribution to maritime security, requires changes in the constitution to enable naval personnel to exercise law enforcement authority or to employ military weapons to this end. Appropriate proposals are being made.
\end{abstract}

Keywords: Maritime Sicherheit, Kontrolle Seeraum, Schutz Seeverbindungen, Bündelung der Zuständigkeiten, mögliche Verfassungsänderung

\section{Einführung: Sicherheit heute}

$\mathrm{D}$ ie aktuelle Debatte um die innere Sicherheit Deutschlands hat viele Facetten. Einer der Brennpunkte der Diskussion sind dabei etwaige verfassungsrechtliche Änderungen zur Erweiterung von Befugnissen und Handlungskompetenzen der Streitkräfte. In dieser Diskussion ist das Thema der maritimen Sicherheit nur sporadisch beleuchtet worden, obwohl gerade die maritime Sicherheit von essentieller Bedeutung für Deutschland ist. Auch hier geht es wesentlich um die Frage der Notwendigkeit einer Erweiterung der Handlungskompetenzen der Streitkräfte, insbesondere der Marine, zur subsidiären Unterstützung anderer Vollzugsorgane in den Fällen, in denen diese mangels entsprechender Mittel und Fähigkeiten nicht tätig werden können.

Vor diesem Hintergrund zielt dieser Aufsatz darauf ab, das Thema »maritime Sicherheit « stärker ins öffentliche Bewusstsein zu rücken. Ausgehend von einer kurzen Bestandsaufnahme zur sicherheitspolitischen Lage und zur Bedeutung der maritimen Dimension für Deutschlands Sicherheit wird der Handlungsbedarf aus operativer Sicht unter Berücksichtigung internationaler Initiativen skizziert, um sich dann im Schwerpunkt den rechtlichen Implikationen der Thematik zu widmen und Vorschläge zur Änderung der verfassungsrechtlichen Grundlagen aus maritimer Sicht abzuleiten.

Die Risiken für unsere Sicherheit unterscheiden sich heute signifikant von denen der Vergangenheit, was wiederum bedeutet, dass bisher bewährte Strategien und Vorgehensweisen auf den Prüfstand zu stellen sind, da sie nicht zwangsläufig auch für die Zukunft taugen.

Noch vor zwei Dekaden definierte sich Sicherheit unter den Vorzeichen des Ost-West-Konflikts vorrangig in militärischen Kategorien. Nachdem sich der Pulverqualm des Ost-WestKonflikts mit all seinen schablonenhaften, aber doch berechenbaren Perzeptionen verzogen hatte, sind ganz neue

\footnotetext{
* Kapitän zur See Rainer Brinkmann ist stellvertretender Kommandeur und Chef des Stabes Einsatzflottille 1 in Kiel.

** Fregattenkapitän und Assessor iur. Dirk Peters, LL.M. (George Washington University, USA) ist Referent für Völkerrecht, Seerecht und Havariewesen im Bundesverteidigungsministerium.
}

Herausforderungen deutlich geworden. Regionale Konflikte, Klimawandel, Migration, Energieversorgung, Rohstoff- und Wasserknappheit, die Erosion staatlicher Macht in der Dritten Welt, der Machtzuwachs nichtstaatlicher Akteure und der Konzerne, Armut und Überbevölkerung kennzeichnen schlagwortartig Risiken, aus denen Konfliktpotential erwächst und die zunehmend an Aktualität gewinnen.

Mit diesen Risiken gehen außerdem markante makroökonomische und geopolitische Entwicklungen einher, die absehbar das Leben der Menschheit in der nächsten Generation verändern werden. Hierzu zählt bspw. die Entstehung neuer wirtschaftlicher und politischer Gravitationszentren in Asien bei gleichzeitiger Überalterung der westlichen Gesellschaften mit all den Konsequenzen für deren Sozialsysteme und Staatsfinanzen. Alle diese Entwicklungen vollziehen sich nicht unabhängig voneinander, sondern bedingen und beeinflussen sich wechselseitig. Das Weißbuch der Bundesregierung spricht demzufolge auch von vernetzter Sicherheit. Die diffuse Melange von Konfliktpotentialen und Risiken entlädt sich in den unterschiedlichsten Formen und Eruptionen, die das friedliche Miteinander gefährden.

Die derzeit unmittelbarste Bedrohung westlicher Sicherheit geht zweifelsohne von dem religiös motivierten, anti-westlichen Terrorismus, der Proliferation von Waffen und zugehöriger Technologie und der organisierten Kriminalität einschließlich des Menschen- und Drogenhandels aus. Dabei werden die Weltoffenheit des Westens, die sich globalisierende Wirtschaft und die zunehmenden internationalen Abhängigkeiten und Verflechtungen mehr und mehr zur Achillesferse westlicher Sicherheit. Der 11. September 2001 und mögliche andere Horrorszenarien lehren, dass die Ausmaße terroristischer Anschläge denen von klassischen militärischen Auseinandersetzungen sehr nahe kommen und das empfindliche inner- und zwischenstaatliche Gleichgewicht in vielerlei Hinsicht nachhaltig stören können: Börsenkurse brechen ein, Ölpreise explodieren, der internationale Handel stagniert.

Vor dem Hintergrund der sich mehr und mehr verzahnenden inneren und äußeren Sicherheit wird eine vorbeugend angelegte, ressortübergreifende und international eingebettete 
Sicherheitspolitik notwendig, in der sich militärische, polizeiliche, wirtschaftliche, entwicklungspolitische, diplomatische und humanitäre Handlungsoptionen komplementieren. Die sicherheitspolitische Situation gebietet es, die streng reglementierten Handlungsmöglichkeiten des Staates zur Risikovorsorge und Krisenbewältigung zu überprüfen und ggf. zu adaptieren. In diesem sicherheitspolitischen Kontext gewinnt die maritime Dimension aus unterschiedlichen Gründen zunehmend an Bedeutung.

\section{Sicherheit und See}

\subsection{Die Bedeutung der See für die Sicherheit Deutschlands}

Es sind vor allem volkswirtschaftliche, rechtliche, demographische und sicherheitspolitische Gründe, die die besondere Bedeutung der See für Deutschlands Sicherheit bedingen. Deutschland ist Exportweltmeister und mehr als irgendein anderer westlicher Industriestaat auf einen ungehinderten weltweiten Handel angewiesen. Deutschlands Prosperität hängt unmittelbar vom freien Welthandel und dem Zugang zu den weltweiten Märkten ab. Und dieser Handel und Warenverkehr spielt sich mit Masse auf und über See ab, was die ungehinderte Nutzung der See und den maritimen Raum generell in den Fokus deutscher Sicherheitsinteressen rücken lässt.

Deutschlands Verhältnis zur und Abhängigkeit von der See drückt sich wie folgt in Zahlen aus ${ }^{1}$ : Eine halbe Million Menschen sind in Deutschland in der maritimen Wirtschaft beschäftigt. Deutsche Eigner unterhalten die drittgrößte Handelsflotte der Welt; bei den Containerschiffen rangiert Deutschland auf Platz 1: Weltweit wird fast jedes dritte Containerschiff von deutschen Reedern betrieben. 95\% des weltweiten Güterfernverkehrs und 90\% des Außenhandels der EU werden über See abgewickelt. Hamburg und Bremen zählen neben Rotterdam und Antwerpen im Containerumschlag zu den vier leistungsstärksten europäischen Häfen. 139.000 Schiffe laufen jährlich deutsche Häfen an. Laut einer Studie des Hamburger Weltwirtschaftsinstituts (HWWI) wird der Containerumschlag bis 2030 weltweit um nicht weniger als $620 \%$ steigen.

Das Güteraufkommen der deutschen Seehäfen wird bis 2025 um das Eineinhalbfache zunehmen, Container-, Fähr- und Ro/Ro-Verkehr ${ }^{2}$ werden sich verdreifachen. Deutschlands Abhängigkeit von einer sicheren Rohstoffzufuhr ist besonders markant. Bei Energierohstoffen ist Deutschland zu 71,3\% von Importen abhängig, bei metallischen Rohstoffen zu 93,8\%, bei Edelmetallen zu $98 \%$ und bei Nichtmetallen zu fast $100 \%$. Rohöl wird zu $97 \%$ eingeführt, Erdgas zu $85 \%$ und Steinkohle zu $67 \%$, in der Masse auf dem Seeweg.

Die deutsche Werftindustrie bekleidet unter den Schiffsbaunationen aus technologischer Sicht eine Spitzenstellung; im

\footnotetext{
1 Hamburger Weltwirtschaftsinstitut (HWWI), Schriftenreihe Strategie 2030 - Maritime Wirtschaft und Transportlogistik, September 2006. 2 Ro/Ro-Verkehr: roll-on roll-off traffic.
}

Spezialschiffbau ist Deutschland trotz der Konkurrenz aus Fernost noch führend.

Die See ist aber nicht nur als Drehscheibe des Handels von Bedeutung. Mehr und mehr rückt die Erschließung von Rohstoffen und Energiequellen im maritimen Raum in den Vordergrund. Die jüngst artikulierten territorialen Ansprüche Russlands, Kanadas und Dänemarks auf den Meeresgrund unterhalb des Polareises illustrieren diese Entwicklung. Oft wird vergessen, dass die Nordsee nach Russland, den USA und Saudi-Arabien die viertgrößte Quelle fossiler Energieträger ist.

Die für die Nutzung der Meere und die Erschließung von für Nahrung, Energieträger und Rohstoffe im maritimen Raum notwendigen Technologien sind für den Industrie- und Technologiestandort Deutschland höchst interessante Innovations- und Investitionsfelder.

Mit der wirtschaftlichen Nutzung des maritimen Raumes geht eine andere Entwicklung einher. Da die Masse des weltweiten Handels über See abgewickelt wird, bilden sich an den Abgangs- und Bestimmungshäfen des Seeverkehrs immer komplexere Ballungszentren aus. Schon heute leben 2/3 der Weltbevölkerung in Küstenstreifen von weniger als 100 Kilometer Tiefe. Dieser Trend nimmt zu. Die Ballung von Bevölkerung und maritimer Infrastruktur im küstennahen Raum hat per se einen sicherheitspolitischen Impetus. Denn Krisen entstehen nun aber einmal dort, wo Menschen leben, wo sie arbeiten und wo sie miteinander hadern. Und das ist der maritime Raum. Ballungszentren sind das bevorzugte Ziel terroristischer Aktivitäten, da sich dort die "größtmögliche Wirkung « und so die größtmögliche Verunsicherung der Bevölkerung erzielen lässt.

Der maritime Raum gewinnt aber nicht nur als Arena krisenhafter Entwicklungen und terroristischer Anschläge sicherheitspolitisch an Bedeutung, sondern wegen des besonderen Rechtsstatus der Hohen See auch als Bühne staatlichen Handelns im Rahmen von Konfliktverhütung und Krisenmanagement. Die Hohe See ist ein hoheitsfreier Raum, in dem nationalstaatliche Rechtsregime nicht gelten. Die Hohe See ist »Brücke zwischen den Kontinenten « und erlaubt es der Politik, militärisches Handeln über See zu entfalten und politischen Willen deutlich zu machen, wo dies zur Wahrung eigener Interessen geboten ist. Durch die Nutzung der Hohen See als Basis und Drehscheibe politischen Handelns und militärischer Machtentfaltung werden nationalstaatliche Interessen von Drittstaaten im rechtlichen Sinne nicht berührt oder verletzt. Dies unterscheidet maritime von land- oder luftgestützten Operationen.

Dies alles macht die See gleichermaßen zu einem schutzbedürftigen Raum für die eigene ungehinderte Nutzung und zum Schauplatz von Aktivitäten, die unsere Sicherheit gefährden, wie z.B. Proliferation, Schmuggel, Piraterie, Migration, Umweltverschmutzung und Menschenhandel. 


\subsection{Gefahren, Bedrohungen und Risiken im maritimen Raum}

Die den maritimen Raum betreffenden Gefahren und Risiken werden grundsätzlich den Bereichen Safety und Security zugeordnet. Safety meint dabei im Wesentlichen die nautische und betriebliche Sicherheit für Seeverkehr und maritime Infrastruktur. Security hingegen bezeichnet die Sicherheit vor rechtswidrigem Handeln, Kriminalität und intendierten Anschlägen und Angriffen auf Seeverkehr und maritime Infrastruktur. Zu diesem Komplex zählt der Terrorismus ebenso wie die Piraterie, die Proliferation, der Drogenschmuggel und der Menschenhandel. Zwar verlangen Dichte, Aufkommen und Komplexität des Seeverkehrs den für die betriebliche und nautische Sicherheit verantwortlichen Behörden weitere Anstrengungen ab (safety), es sind aber vor allem die der Security entgegenstehenden Risiken, die eine besondere Herausforderung darstellen und die Frage nach einem Beitrag des Militärs aufwerfen.

Es ist nur wenig Phantasie vonnöten, sich Horrorszenarien vorzustellen, bei denen die mögliche immense Wirkung terroristischer Anschläge im maritimen Raum deutlich wird: sei es, dass ein im Küstenvorfeld havarierter Gas- oder Öltanker eine Umweltkatastrophe gewaltigen Ausmaßes auszulösen imstande ist, sei es, dass das Kapern eines Kreuzfahrers eine menschliche Tragödie nach sich ziehen kann oder sei es, dass ein Anschlag auf das Steuerungszentrum eines Containerhafens den Seehandel nachhaltig beeinträchtigen kann.

Aber es geht nicht nur um die Verhinderung derartiger Horrorszenarien in den der deutschen Küste vorgelagerten Seegebieten. Deutschland ist mit der Unterzeichnung des Seerechtsübereinkommens von 1982 international Verpflichtungen eingegangen (z.B. Bekämpfung der Piraterie), die es gegenwärtig außerstande ist, zu erfüllen. Diese Misere erfahren die am Horn von Afrika im Rahmen der Operation Enduring Freedom eingesetzten Marineeinheiten täglich aufs Neue. Dort ist die Marine auf der Grundlage des Artikels 24 GG (System kollektiver Sicherheit) legitimiert, sich am Kampf gegen den internationalen Terrorismus zu beteiligen. Sie ist hingegen nicht befugt, gegen Piraterie oder Waffenhandel vorzugehen. Was also ist zu tun, um die maritime Sicherheit zu gewährleisten?

\subsection{Handlungsbedarf - eine erste Annäherung}

Da Ort, Zeit und Form terroristischer Anschläge nur schwerlich vorhersehbar sind, kommt der Überwachung und Aufklärung im maritimen Raum zwecks Prävention eine herausragende Bedeutung zu. Was im Luftverkehr bereits seit langem praktiziert wird, nämlich ein umfassendes Überwachungsregime des gesamten Luftverkehrs, tut auch für den maritimen Raum Not. Nur mittels eines rigiden Lagebildes lassen sich Anomalien und damit Risiken frühzeitig erkennen. Hat sich aber eine Bedrohung erst einmal konkretisiert, bedarf es geeigneter Mittel, um reagieren zu können und dieser Bedrohung Herr zu werden. Beide Probleme, die Prävention ebenso wie die Reaktion, werfen dann wiederum Fragen der Mittelausstattung, der Kompetenzen und der Kooperation der Vollzugsbehörden und der Streitkräfte auf. Auf eine kurze Formel gebracht, besteht das Dilemma maßgeblich darin, dass einerseits diejenigen polizeilichen Vollzugsbehörden, die für die Gefahrenabwehr zuständig sind, nicht über die Mittel und Fähigkeiten verfügen, andererseits die Streitkräfte über die Fähigkeiten verfügen, rechtlich aber keine Befugnisse haben.

Ein weiterer Aspekt kommt hinzu: Gerade wegen der weltumspannenden und grenzüberschreitenden Dimension maritimer Sicherheit sind einzelstaatliche Anstrengungen wenngleich vonnöten, so doch nur wenig erfolgversprechend. Es bedarf international eines konzertierten und abgestimmten Handelns. Einem solchen Handeln müssen aber einzel- und innerstaatliche Anstrengungen vorausgehen, um die notwendigen Voraussetzungen einer umfassenden Kooperation zu schaffen. Behördliche Zuständigkeiten, Strukturen, Kompetenzen und Verfahren erweisen sich als überkommen und wenig zweckdienlich. Der Notwendigkeit von Reformen stehen rechtliche und grundgesetzliche Bestimmungen, föderale Strukturen, aber auch Partikularinteressen der jeweiligen staatlichen Akteure entgegen, die um Einfluss und Kompetenzen fürchten.

In Anbetracht einer Vielzahl internationaler Initiativen wird jedoch der Druck auf Deutschland zunehmen. Es wird kaum möglich sein, sich diesen Entwicklungen zu entziehen, so dass weitere Reformen und u.U. auch grundgesetzliche Änderungen unerlässlich sind. Um nicht vor vollendete Tatsachen gestellt zu werden, sollten diese Prozesse und Entwicklungen aktiv mitgestaltet werden. Nicht zuletzt kann eine aktive Beteiligung dazu beitragen, die Initiativen zu bündeln und $\mathrm{zu}$ integrieren und Duplizierungen zu vermeiden.

\section{Internationale Initiativen ${ }^{3}$}

Abseits des in Umfang und Anspruch begrenzten Projekts »FRONTEX «, das darauf zielt, die polizeiliche Zusammenarbeit in der EU zur Sicherung der Außengrenzen und zur Verhinderung illegaler Einwanderung zu verbessern, sind der Öffentlichkeit kaum weitere Initiativen bekannt. Ganzheitliche Ansätze zum Thema maritime Sicherheit werden gegenwärtig vor allem im militärischen Bereich diskutiert. Fünf Initiativen sind dabei von besonderer Bedeutung:

\subsection{Maritime Security Operations (MSO)}

Das informelle Forum der Oberbefehlshaber der europäischen Marinen $\left(\mathrm{CHEN}^{4}\right)$ hat in diesem Jahr ein Strategiepapier ${ }^{5}$ entwickelt, das den Anstoß zur Formulierung einer gesamteuropäischen, ressortübergreifenden Strategie geben soll. Ausgangspunkt war die Lagebewertung, dass vielfältige Bedro-

\footnotetext{
3 Der von der »Interparliamentary European Security and Defence Assembly der WEU (v. 06.12.2005) veröffentlichte Bericht Surveillance of the maritime and coastal areas of European states (Document A/1920) gibt einen guten Überblick über die in den drei EU Säulen (Europäische Gemeinschaft - GASP - Polizei und Justiz) laufenden Maritime Security Operations Initiativen. 4 Chiefs of European Navies (CHEN).

5 CHEN, Developing a European Interagency Strategy for Maritime Security Operations - a naval view (MSO), 2007; vgl. hierzu die Kommentierung des Grünbuches Meerespolitik durch die CHEN 05/2007.
} 
hungen die Sicherheit und wirtschaftliche Stabilität Europas gefährden und die Handlungsoptionen eines einzelnen Staates nicht ausreichen, um diesen zu begegnen. In dieser Strategie wird ausdrücklich die Rolle der bestehenden nationalen und multinationalen Strukturen und Organisationen bei der Ausgestaltung der Strategie betont und die Notwendigkeit der Berücksichtigung nationaler Besonderheiten bestätigt.

Das Strategiepapier beschreibt den Handlungsbedarf in vier Entwicklungslinien:

- die zwischenstaatliche Zusammenarbeit, die Kooperation mit betroffenen Organisationen sowie die Klärung juristischer Fragen,

- die Ausgestaltung des Informationsaustausches und Lagebildaufbaus,

- die Einbindung der Streitkräfte,

- die Einbindung der zivilen Schifffahrt.

Das Pendant zur europäischen Initiative ist der von der US Navy gegebene Denkanstoß, die Relevanz des maritimen Raums für die Sicherheit intensiver zu reflektieren:

\subsection{Maritime Domain Awareness (MDA) - Maritime Situational Awareness}

Das »Concept for Alliance and Coalition Maritime Domain Awareness (MDA) ist ein »White Paper« des US MoD ${ }^{6}$, welches den unterschiedlichen NATO-Gremien bereits Anfang 2006 mit dem Ziel vorgestellt wurde, eine Diskussion innerhalb der NATO über das Thema MDA zu beginnen.

Der Begriff der Maritime Domain (MD) bezieht sich dabei auf alle Aktivitäten, Geschehnisse und Planungen mit Bezug zur See: Vorgänge auf und unter Wasser, in Relation zu oder angrenzend an Meere, befahrbare Wasserstraßen etc. MDA ist das resultierende Verständnis für alle Sicherheitsaspekte (security \& safety) im maritimen Raum, die Wirtschaft, Infrastruktur oder Umwelt beeinträchtigen könnten. Das Konzept legt den Schwerpunkt auf die Entwicklung eines Lagebewusstseins (situational awareness). Dieses soll die Grundlage zur Entwicklung von Fähigkeiten zur Prävention, Abschreckung und Verhinderung von Terror und kriminellen Handlungen sein. Die Integration bestehender nationaler Systeme zur Lagebilderstellung wird grundsätzlich einer Neuentwicklung vorgezogen.

Ziel ist es, staatliche Entscheidungsträger mit validen Informationen zu versorgen, die die Ableitung von Handlungsoptionen zulassen. MDA verfolgt einen ganzheitlichen, globalen Ansatz und stützt sich auf Ressourcen und Fähigkeiten beteiligter Nationen.

\section{Als Hauptaufgaben formuliert das MDA-Konzept:}

- die kontinuierliche Überwachung u.a. von Schifffahrt, Ladungen, Besatzungen und Passagieren,

- den Zugang zu Fahrzeug-, Einrichtungs- und Infrastrukturdaten,

6 Office of the Under Secretary of Defense (Acquisition, Technology \& Logistics) International Cooperation.
- die Sammlung und Analyse von Daten und deren Verbreitung bei entsprechenden Entscheidungsträgern,

- die Entwicklung von und den Zugang zu Datenbanken.

\subsection{Virtual Regional Maritime Traffic Center}

Verschiedene pragmatische Ansätze der konzeptionell-strategischen Überlegungen der europäischen und amerikanischen Streitkräfte sind komplementär. Vor dem Hintergrund, dass sich das Mittelmeer in der zurückliegenden Dekade zunehmend zur Drehscheibe von illegaler Einwanderung, Waffenschmuggel und organisierter Kriminalität entwickelte, wurde unter Federführung der italienischen Marine ein regionales Überwachungssystem implementiert, das darauf zielt, alle relevanten Informationen über den Schiffsverkehr im Mittelmeer in ein umfassendes Lagebild zu integrieren. Dieses Lagebild bezieht neben Informationen, die von Marineeinheiten gesammelt werden, auch AIS-Daten ${ }^{7}$ ein. Die Deutsche Marine unterstützt dieses Projekt im Rahmen ihrer Möglichkeiten.

\subsection{NATO: Maritime Safety and Security Information System (MSSIS)}

Bei dem Maritime Safety and Security Information System (MSSIS) handelt es sich um ein von der NATO entwickeltes Instrument, mit dessen Hilfe ein Lagebild auf See erstellt werden kann. MSSIS stützt sich dabei auf von nunmehr 28 angeschlossenen Staaten bereitgestellte Lageinformationen, die mit Hilfe einer speziellen Software zu einem Gesamtlagebild aggregiert werden. MSSIS hat es ermöglicht, nach gründlicher Analyse der bereitgestellten Rohdaten unter Einsatz von Algorithmen und anderen Auswertemechanismen die Zahl der erforderlichen physischen Untersuchungen (»Boardings «) verdächtiger Schiffe im Rahmen der Operation Active Endeavor im Mittelmeer um mehr als die Hälfte zu reduzieren. Da sich MSSIS wesentlich auf AIS Informationen abstützt, die manipulierbar sind und die bislang keine Seefahrzeuge unter 300 BRT erfassen, bedarf dieses System der Weiterentwicklung und Ergänzung. Zwar validieren manche Staaten diese Daten bereits durch digitale Radarbilder, die Masse der Lageinformationen wird allerdings noch nicht verifiziert. Anders als bei marinetypischen Operationen üblich, sind die im Lagebild enthaltenen Informationen auch nicht zuverlässig. Gleichwohl ist MSSIS ein wichtiges Werkzeug, das es im Rahmen weiterer Anstrengungen auszubauen und mit anderen Systemen zu vernetzen gilt.

\subsection{European Defence Agency: Maritime Surveillance}

Die European Defence Agency, die dem Hohen Vertreter der Gemeinsamen Außen- und Sicherheitspolitik (GASP) der EU unmittelbar untersteht, hat unterschiedliche Projekte aufge-

\footnotetext{
7 AIS: Automatic Identification System; System auf Schiffen zum gegenseitigen Austausch von Lagedaten zur Kollisionsverhütung.
} 
legt, die die länder- und ressortübergreifende Zusammenarbeit, den Informationsaustausch, die technische Standardisierung und etwaige gemeinsame Beschaffungen befördern sollen. Vorrangiges Ziel ist, die maritimen Lagebilder der Staaten zu verdichten, zu vernetzen und den Informationsaustausch zu intensivieren.

\section{Nationale Initiativen - Küstenwache und Maritimes Sicherheitszentrum}

Das Plädoyer, sich intensiver mit dem Thema maritimer Sicherheit zu befassen, sollte aber nicht darüber hinwegtäuschen, dass auch in Deutschland in den letzten zehn Jahren durchaus Fortschritte erzielt worden sind. Um die Effektivität bei der Sicherung und Kontrolle des Seeverkehrs, der Katastrophenabwehr und der Prävention und Verfolgung von Straftaten im eigenen Zuständigkeitsbereich zu verbessern, wurden die Vollzugsbehörden des Bundes 1994 zum sogenannten Koordinierungsverbund Küstenwache zusammengeschlossen, dem die Bundespolizei, der Zoll, die Wasser- und Schifffahrtsverwaltung, der Fischereischutz und das Bundesamt für Seeschifffahrt und Hydrographie angehören. Nach wie vor aber liegt die schifffahrtspolizeiliche Zuständigkeit im Küstenmeer bei den fünf Küstenländern mit ihren Wasserschutzpolizeien. In den Verbund der Küstenwache sind die Wasserschutzpolizeien nicht integriert.

Ein weiterer innovativer Schritt, der - sofern die damit verbundenen Chancen auch genutzt werden - auch international Modellcharakter haben kann, ist die Errichtung des sogenannten Maritimen Sicherheitszentrums in Cuxhaven. Mit dem Maritimen Sicherheitszentrum wird ein Koordinierungszentrum derjenigen Behörden und Organisationen des Bundes und der Länder geschaffen, die u.a. für maritime Sicherheit zuständig sind. Erstmalig ist auch die Marine mit einem Verbindungselement dort vertreten (Havariekommando, Wasser- und Schifffahrtsverwaltung des Bundes, Wasserschutzpolizeien, Bundespolizei, Zoll, Fischereischutz und Flottenkommando).

Den operativen Nukleus des MSZ bildet das Gemeinsame Lagezentrum See (GLZ-See). Dort werden auf der Grundlage unveränderter Zuständigkeiten Informationen aller Vollzugsbehörden gebündelt und Einsätze koordiniert. Das ist leichter gesagt als getan. Allein der Austausch und Umgang mit teilweise sensiblen Informationen wirft viele Fragen und datenschutzrechtliche Probleme auf, die noch einer klaren Regelung bedürfen.

Modellcharakter kann das MSZ aber gerade deswegen auch für andere Staaten haben, weil die Idee nicht an den Grundfesten innerstaatlicher Zuständigkeiten rührt, sondern lediglich die Zusammenarbeit der Vollzugsbehörden optimiert und auf eine neue Grundlage stellt.

Eine Einbindung der Deutschen Marine, die über den bislang gültigen Rahmen der Zusammenarbeit mit den Vollzugsbehörden hinausgeht (Amtshilfe/technisch-logistische Unterstützung), kann nur über ein zukünftiges Seesicherheitsgesetz und eine damit verbundene Verfassungsänderung erfolgen.
Die Notwendigkeit der Einbindung der Marine leitet sich aus operativen Gründen ab.

\subsection{Der Beitrag der Marine - die operative Dimension}

Wie bereits erwähnt, ist die conditio sine qua non einer erfolgversprechenden Früherkennung und Abwehr von Bedrohungen im maritimen Raum ein umfassendes Lagebild, in das alle relevanten und verfügbaren Informationen und Aufklärungsergebnisse einfließen. Wegen des weltweiten Seeverkehrs und der internationalen Verflechtungen ist ein solches Lagebild nur im Rahmen internationaler Kooperation realisierbar. Anknüpfend an die Initiativen von EDA und CHEN wäre in einem ersten Schritt die Vernetzung vorhandener Lagezentren anzustreben und ein anlassbezogener Informationsaustausch vorzusehen.

Da das Geschehen in der Ostsee für Mittelmeeranrainer nicht in gleicher Weise interessant ist wie für die Staaten des Ostseebereichs, sollte die notwendige Netzwerkbildung in regional begrenztem Kontext und unter Nutzung der bereits vorhandenen Überwachungssysteme erfolgen. Das Rad muss ja nicht einmal mehr neu erfunden werden. Geographisch gesehen bieten sich in Bezug auf den europäischen Festlandssockel Subsysteme für die Ostsee, die Nordsee, die irische See und den englischen Kanal, die iberische Halbinsel sowie das Mittelmeer an. Die Grundidee kommt dabei letztlich der eines Puzzles gleich: regionale Subsysteme ergeben in der Summe ein quasi weltumspannendes Lagebild.

Die Marine kann zu einem solchen Lagebild und zu einem solchen Informationsverbund substantiell beitragen. Ihre Einheiten operieren weltweit, verfügen über vielfältige Aufklärungsmittel und ausgezeichnete Kommunikationsmöglichkeiten, die eine Datenübermittlung in Echtzeit ermöglichen. Militärisch werden auch Seegebiete überwacht, in die »Auge und Arm « anderer Vollzugsbehörden nicht reichen, in denen aber Marineeinheiten operieren. Die militärischen Hauptquartiere verfügen darüber hinaus regelmäßig bereits über ein recht umfassendes Lagebild, zumal ihr originärer Auftrag - die Abwehr äußerer Gefahren und der Schutz des Territoriums - ein solches Lagebild qua Verfassungsauftrag notwendig macht. Überdies sind die europäischen maritimen Hauptquartiere bereits weitgehend vernetzt und der Austausch militärisch relevanter Informationen ist praktizierte Normalität.

In der Kooperation der Marine mit dem Maritimen Sicherheitszentrums dürfte vor diesem Hintergrund einer der Schlüssel zum Erfolg liegen. Die Marine wird auf der Grundlage des im militärischen Hauptquartier geführten Lagebildes die im MSZ verfügbaren ressortspezifischen Daten anderer Vollzugsbehörden ergänzen können.

Im Übrigen muss auch der Dialog zwischen den Vollzugsbehörden und der Marine einerseits und der Seewirtschaft andererseits angestoßen werden. Es sollte im ureigensten Interesse der Seewirtschaft und der Reedereien mit ihren weltweit präsenten Schiffen liegen, einen substantiellen Beitrag zu einem umfassenden Lagebild zu leisten, das dann die staat- 
lichen Behörden in die Lage versetzt, Vorsorge bei erkannten Anomalien oder Bedrohungen zu treffen. Ein Verbund aus behördlichen, militärischen und seewirtschaftlichen Aufklärungsbeiträgen kann die Qualität des maritimen Lagebildes erheblich verbessern.

Für den Fall, dass sich Verdachtsmomente hinsichtlich Unregelmäßigkeiten, Gefährdungen der Sicherheit oder geplanter Anschläge häufen oder konkretisieren, verfügt die Marine ebenfalls über ein breites Spektrum an Fähigkeiten, mit denen die zuständigen Vollzugsbehörden bei der Gefahrenabwehr subsidiär unterstützt werden könnten, falls sie mit eigenen Mitteln hierzu allein nicht in der Lage sind. Eine solche subsidiäre Unterstützung durch die Streitkräfte erfordert allerdings die Änderung der Rechtslage, nachdem das Bundesverfassungsgericht in seinem Urteil zum Luftsicherheitsgesetz klargestellt hat, dass der Einsatz spezifisch militärischer Mittel zur Unterstützung der zuständigen Behörden bei Abwehr und Bewältigung schwerer Unglücksfälle nicht statthaft ist. Auch im Rahmen des bereits rechtlich Möglichen gibt es Handlungsbedarf: So bestehen bspw. bis dato keine abhörsicheren Kommunikationsverbindungen zwischen den Seestreitkräften und den übrigen Vollzugsbehörden auf See. Ferner mangelt es an geeigneten Aussetzungsvorrichtungen für Speedboote der Bundespolizei auf Einheiten der Marine u.ä. Bei technischen Problemen dieser Art fehlt es derzeit vor allem deshalb an zielführenden Lösungen, weil weder den Streitkräften noch den Vollzugsbehörden finanzielle Mittel für die Ausgestaltung der gegenseitigen Zusammenarbeit zur Verfügung stehen. Dabei wird in der Bevölkerung im Ernstfall niemand Verständnis haben, wenn eine Operation zur Gefahrenabwehr daran scheitert, dass keine gemeinsamen Kommunikationsmittel zur Verfügung standen bzw. Einsatzkräfte der Polizei aufgrund technisch unterschiedlicher Standards nicht von der Marine verbracht werden konnten.

Für die Institutionalisierung der Zusammenarbeit von Vollzugsbehörden und Streitkräften ist es erforderlich, dass ein konzertiertes Vorgehen zur Abwendung eines Anschlags oder Schadensfalls periodisch erprobt wird. Hierzu bedarf es gemeinsamer Ausbildungsmaßnahmen, Übungen und Verfahren. Was nicht geübt wird, wird im Ernstfall kaum gelingen.

Der Beitrag der Marine zu einem umfassenden maritimen Lagebild und die Erweiterung der Handlungskompetenzen der Marine im Fall drohender oder tatsächlicher schwerer Unglücksfälle sollten aber nicht über ein anderes ungelöstes Problem hinwegtäuschen, das bereits angesprochen wurde. Deutschland ist mit Unterzeichnung des Seerechtsübereinkommens Verpflichtungen eingegangen, die es gegenwärtig außerstande ist zu erfüllen. Für den Kampf gegen die Piraterie, den Drogenhandel, den Menschenschmuggel und die Proliferation auf hoher See ist die zuständige Bundespolizei nicht gerüstet, während die Marine durchaus über die dafür notwendigen Fähigkeiten, nicht aber die Befugnisse verfügt.

Was darf nach geltender Rechtslage aber die Marine nun eigentlich? Es ist an der Zeit, sich der rechtlichen Situation zuzuwenden.

\subsection{Der Beitrag der Marine - Die rechtliche Dimension}

\section{Spezifizierung der Problemstellung}

Nach der verfassungsrechtlichen Kompetenzverteilung obliegt die Gewährleistung der inneren Sicherheit der Polizei, die äußere Sicherheit wird durch die Streitkräfte gewährleistet. Die Trennung von äußerer und innerer Sicherheit und die damit verbundene grundsätzliche Trennung polizeilicher und militärischer Aufgaben ist nicht das Ergebnis eines bloßen Zufalls, sondern war von den Verfassungsgebern wohl überlegt, als sie 1956 die Wehrverfassung ins Grundgesetz integrierten. ${ }^{8}$ Vor dem Hintergrund der historischen Erfahrungen entschied man sich ganz bewusst dafür, Errichtung und Auftrag der Streitkräfte nicht in einem einfachen Fachgesetz, sondern in der Verfassung selbst zu regeln. ${ }^{9}$ Mit Einführung der Notstandsverfassung 1968 wurde der Einsatz der Streitkräfte zu Polizeizwecken erstmals auf eine verfassungsrechtliche Grundlage gestellt, der Grundsatz der strikten Aufgabentrennung jedoch beibehalten. Eine Veränderung dieses Grundsatzes erfordert daher einen Eingriff in die Verfassung selbst. ${ }^{10}$

Die Frage nach dem gesetzlichen Regelungsbedarf bei der Äußeren und der Inneren Sicherheit berührt zentral den verfassungsmäßigen Aufgabenzuschnitt der Streitkräfte. Der Koalitionsvertrag vom 11. November 2005 enthält hierzu lediglich eine hinhaltende Aussage. Dort heißt es: »Neben der Teilnahme an der internationalen Konfliktbewältigung bleibt die Landesverteidigung unter veränderten Bedingungen und Aufgabenstellungen der verfassungsmäßige Kernauftrag der Bundeswehr. Gerade im Hinblick auf asymmetrische Formen der Bedrohung, die insbesondere aus terroristischen Aktivitäten bestehen, ist die äußere von der inneren Sicherheit nicht mehr trennscharf zu unterscheiden. Soweit für besondere Gefährdungen der Sicherheit unseres Landes gesetzlicher oder verfassungsmäßiger Regelungsbedarf besteht, wird die Bundesregierung Initiativen vorlegen.«

Es ist nun Sache des Gesetzgebers, einen zukunftsfähigen Aufgabenrahmen für die Streitkräfte in der Verfassung bereitzustellen. Unter dem Gesichtspunkt der Rechtssicherheit für das Handeln der Streitkräfte besteht verfassungsrechtlicher Regelungsbedarf, denn die Angriffe des 11. September 2001 und die Anschläge in London und Madrid haben der westlichen Welt in aller Deutlichkeit vor Augen geführt, wie wenig ihre etablierten Sicherheits- und Verteidigungssysteme geeignet sind, hinreichenden Schutz gegen asymmetrische Bedrohungen zu bieten. Die klassischen Grenzen zwischen einem von außen herangetragenem Krieg und innerstaatlicher polizeilicher Gefahrenabwehr verschwimmen. Die Bundeswehr übernimmt de facto bereits heute klassische Polizeiaufgaben, so zum Beispiel die Absicherung der Küste während des G-8 Gipfels. Die Polizei hingegen benötigt zur Abwehr terroristischer Handlungen militärische Kapazitäten, wenn ein angreifendes Flugzeug abgeschossen oder das Einlaufen eines gefährlichen Schiffes in

8 Soria, DVBl. 2004, 597 m.w.N

9 Edzard Schmidt-Jortzig, Die Öffentliche Verwaltung (DÖV) 2002, 773

10 Vgl. Art. 79 Abs. 1, 2 GG; So auch Wiefelspütz, ZRP 2007, 17 (18). 
einen Hafen im Rahmen der Gewährleistung der Hafensicherheit verhindert werden soll. ${ }^{11}$

Mit der Marine verfügt der Bund über eine weitere Komponente, die praktisch in der Lage ist, Aufgaben des Küstenschutzes zu übernehmen. Wie im Folgenden aufgezeigt wird, ist ein Einsatz der Marine im Innern nach geltender verfassungsrechtlicher Lage nicht zulässig. In Anbetracht dieser neuen Bedrohungen stellt sich die Frage, ob die verfassungsrechtliche Kompetenzverteilung zwischen den Streitkräften und der Polizei aufrechterhalten werden kann. Ein Bedürfnis nach einer rechtlichen Kompetenzerweiterung der Marine ergibt sich daraus, dass sie über Fähigkeiten verfügt, die die Küstenwache nicht oder nicht in vergleichbarem Umfang besitzt. Es besteht mithin eine Diskrepanz zwischen rechtlichem Dürfen und tatsächlichem Können.

\section{Gegenwärtige Rechtslage}

Die Verwendung der Streitkräfte ist im Grundgesetz in verschiedenen Artikeln geregelt. Die in diesem Zusammenhang als maßgeblich zu nennenden Vorschriften sind Artikel 30, 35, 87a, 115a GG. Maßstab für die Beurteilung der Frage nach der Verwendung der Streitkräfte, und damit auch für den Einsatz der Marine, ist Artikel 87a Abs. 2 GG. ${ }^{12}$

\section{a) Zuständigkeit für den Schutz der Territorialgewässer}

Artikel 30 GG regelt die Kompetenzverteilung zwischen Bund und Ländern und legt ein Regel-Ausnahme-Verhältnis fest. Hiernach besitzt der Bund nur die ihm zugewiesenen Kompetenzen, der unbenannte Rest liegt bei den Ländern. ${ }^{13}$ Entweder der Bund oder die Länder sind zuständig; es gibt grundsätzlich keine Doppelzuständigkeit. Art 30 GG ist zwingendes Recht und damit nicht dispositiv, was bedeutet, dass Kompetenzüberlassungen bzw. Kompetenzverschiebungen, die keine Grundlage im Grundgesetz haben, unzulässig sind. ${ }^{14}$ Das Gebiet des Ordnungs- und Polizeirechts obliegt damit den Ländern, so dass der Bund nur punktuell über Gesetzgebungskompetenz verfügt. ${ }^{15}$ Innerhalb der Territorialgewässer sind die Küstenländer für die Wahrnehmung polizeilicher Aufgaben zuständig, der Bund stellt mit Hilfe der Küstenwache die Sicherheit im Schiffsverkehr, den maritimen Umweltschutz und den Fischereischutz sicher. Rechts- und Amtshilfe im Wege der Organleihe ist gemäß Artikel 35 Abs. 1 GG zwischen Bund und Ländern im Bereich der Verwaltung jedoch zulässig. Ein Einsatz der Marine zur Wahrnehmung polizeilicher Aufgaben kommt daher - sofern überhaupt zulässig - im Wege der Organleihe bzw. Amtshilfe in Betracht.

11 Weitere Beispiele bei Waechter, JZ 2007, 61 (62).

12 Vgl. Seifert/Bünker, ThürVBl. 2006, 49 (50).

13 Pieroth, in: Jarass/Pieroth, GG-Kommentar 9. Aufl. 2007, Art. 30 Rn. 1.

14 Pieroth, in: Jarass/Pieroth, GG-Kommentar 9. Aufl. 2007, Art. 30 Rn. 8

15 Eine ausführliche Darstellung der polizeilichen Aufgabenverteilung von Bund und Ländern findet sich bei Papier, DVBl. 1992, $1 \mathrm{ff}$.

\section{b) Ermächtigungsgrundlage zum Einsatz der Streitkräfte}

Die Aufstellung und Einrichtung der Streitkräfte richtet sich nach Artikle 87a Abs. 1 GG: »der Bund stellt Streitkräfte zur Verteidigung auf«. Damit ist unmissverständlich klar gestellt, dass die Grundaufgabe der Bundeswehr die Verteidigung ist. ${ }^{16}$ Auch wenn - bis auf Artikel 87b GG - der Begriff der »Bundeswehr « im Grundgesetz nicht explizit verwendet wird, so ist unbestritten, dass mit den »Streitkräften « nur die Bundeswehr mit ihren Teilstreitkräften und nicht Landes- oder Bundespolizei gemeint ist. Nicht nur die Soldaten als Ganze, sondern auch einzelne Truppenteile oder Material der Bundeswehr sind vom Begriff der Streitkräfte erfasst; denn angesichts moderner Waffentechnik und ihres enormen Wirkpotenzials genügen bereits wenige Soldaten, um typisch militärische Kampfkraft zu projizieren. ${ }^{17}$ Die Verwendung einzelner Soldaten oder die Überlassung typischen Militärgeräts mit dem Ziel, die verfassungsrechtliche Problematik des Streitkräfteeinsatzes im Innern zu umgehen, stellt insofern eine unzulässige Umgehung der Regelungen des Grundgesetzes dar.

Was hingegen unter dem Begriff der Verteidigung zu verstehen ist, bleibt offen und ist dementsprechend umstritten. Nach Artikel 87a Abs. 2 GG dürfen die Streitkräfte außer zur Verteidigung nur eingesetzt werden, soweit es das Grundgesetz ausdrücklich zulässt. Die Regelung ist keine weitere Ermächtigungsgrundlage zum Einsatz der Streitkräfte, sondern begrenzt ihre möglichen Verwendungszwecke im Innern auf die von der Verfassung angesprochen Gefahrenlagen. ${ }^{18}$ Maßnahmen und Handlungen der Streitkräfte, die nicht »Einsätze« im Sinne des Grundgesetzes sind, bedürfen daher keiner besonderen Rechtfertigung. ${ }^{19}$ Um den Bereich der zulässigen Amtshilfe durch die Streitkräfte fassen zu können, ist der Begriff des »Einsatzes « und der »Verteidigung « zu bestimmen.

\section{aa) Der Begriff der Verteidigung}

Einsätze der Streitkräfte sind immer dann zulässig, wenn es sich hierbei um »Verteidigung «i.S.d. Art. 87a Abs. 1 GG handelt. Eine Verteidigung ist eine Handlung, die sich gegen einen Angriff von außen, d.h. außerhalb der Landesgrenzen, richtet. ${ }^{20}$ Nicht erforderlich ist, dass es sich um die Abwehr eines militärischen Angriffs einer fremden Streitmacht handelt. Es reicht aus, wenn der Angreifer über quasi-militärische Strukturen verfügt und der Angriff nach völkerrechtlicher Auslegung den Anwendungsbereich des Art. 51 VN Charta eröffnet, so dass der Angegriffene von seinem Recht auf Selbstverteidigung Gebrauch machen kann. ${ }^{21}$ Bei der in Art. 115a Abs. 1 S. 1 GG enthaltenen Definition des »Verteidigungsfalls«, handelt es sich nach überwiegender Auffassung bloß um einen Unterfall des Verteidigungsbegriffs. Ein Einsatz der Marine zur Überwachung der Küsten, Erstellung eines Lagebildes und selbst zur Abwehr direkter terroristischer Angriffe, beispielsweise auf eine Hafenanlage, ist daher nicht ohne weiteres vom Begriff der »Verteidigung « erfasst. Die Ausdehnung der Verteidigung

16 Schmidt-Jortzig, DÖV 2002, 773

17 Seifert/Bünker, ThürVBl. 2006, 49 (50).

18 Sorai, DVBl 2004, 597 (599) m.w.N.

19 Spranger, NJW 1999, 1003.

20 Pieroth, in: Jarass/Pieroth, GG-Kommentar 9. Aufl. 2007, Art. 87a GG Rn. 9.

21 Baldus, NVwZ 2004, 1278 (1282). 
auf jede Art von Gewalteinwirkung würde anderenfalls den Verfassungsvorbehalt des Art. 87a Abs. 2 GG entwerten und ließe die Grenze zwischen militärischen und polizeilichen Aufgaben verschwimmen. Auch liegt ein (militärischer) Angriff nicht schon deswegen vor, weil nur die Streitkräfte in der Lage sind, ihm wirkungsvoll zu begegnen. Denn aus der faktischen Möglichkeit auf das rechtliche Dürfen zu schließen, ist mit dem Rechtsstaatsprinzip unvereinbar. ${ }^{22}$ Allerdings wird diese Frage - wenn auch nur am Rande - vom BVerfG im Urteil zum Luftsicherheitsgesetz anders beantwortet. ${ }^{23}$

Als ein besonders schwerer Unglücksfall nach Art. 35 II 2 GG könne nach dem allgemeinen Sprachgebrauch unschwer auch ein Ereignis verstanden werden, dessen Eintreten auf den Vorsatz von Menschen zurückgehe. Der Begriff des Unglücksfalls sei weit auszulegen. ${ }^{24}$ Bereits die Vorgänge, die den Eintritt einer Katastrophe mit an Sicherheit grenzender Wahrscheinlichkeit eintreten ließen, sind dafür ausreichend. ${ }^{25}$

Diese Feststellungen des BverfG werden zwar herangezogen, um die Zuständigkeit der Streitkräfte zur Abwehr einer terroristischen Bedrohung zu begründen. Doch zur Abwehr einer terroristischen Bedrohung, die als besonders schwerer Unglücksfall iSd Art. 35 II 2 GG zu werten ist, dürften die Streitkräfte jedoch keine militärspezifischen Waffen einsetzen. Damit wären die Seestreitkräfte dann auf die gleichen Mittel beschränkt, die auch schon den übrigen Vollzugsbehörden zur Verfügung stehen und die sich je nach Bedrohungslage als unzureichend erweisen können.

\section{bb) Der Begriff des Einsatzes}

Wie zuvor festgestellt, bedürfen weder Einsätze zur Verteidigung noch solche, die nicht unter den Begriff des Einsatzes nach Art. 87a Abs. 2 GG fallen, einer verfassungsrechtlichen Prüfung. Der Verfassungsvorbehalt des Art. 87a Abs. 2 GG gilt dementsprechend für Einsätze, die weniger sind als eine »Verteidigung «, aber mehr als ein bloßes »Tätigwerden«. Die in der Literatur hierzu vertretenen Ansichten sind dementsprechend vielfältig. Ein Grundkonsens besteht darin, dass ein Einsatz dann vorliegt, wenn die Bundeswehr als spezifisch militärischer Handlungsverband im hoheitlich-exekutiven Bereich tätig wird. ${ }^{26}$ Ein solches Handeln weist die für staatliches Handeln typisierenden Merkmale der Regelung, Eingriffsmöglichkeit und Zwangsbefugnis auf und kann von einfach hoheitlichem Handeln unterschieden werden. ${ }^{27}$ Ein bloßes »verwenden « der Streitkräfte liegt hingegen vor, wenn es um die Teilnahme an einer Ehrenformation oder die Opfersuche mit Wärmebildkameras geht. Die in Anbetracht der Bedrohungslage erforderlichen Handlungen der Seestreitkräfte unterfallen dem Begriff des Einsatzes und sind aufgrund des Verfassungsvorbehalts des Art. 87a Abs. 2 GG unzulässig. Zur Abwehr entsprechender Bedrohungen wird - wenn auch

22 Effektivitätsgedanken begründen keine automatische Zuständigkeit, vgl. Sattler, NVwZ 2004, 1286.

23 BVerfG, NJW 2006, 751 (754f.); Siehe dazu auch Wiefelspütz, ZRP 2007 Heft 1 m.w.N.

24 BVerfG, NJW 2006, 751, (755); siehe dazu auchWiefelspütz, ZRP 2007 Heft 1. 25 BVerfG, NJW 2006, 751.

26 Schmidt-Jorztig, DÖV 2002, 773 (775); Seifert/Bünker, ThürVBl. 2006, 49 (51). 27 Vgl. den Wortlaut des § 35 VwVfG. subsidiär zu den übrigen Polizeikräften - die Befugnis benötigt, unmittelbaren Zwang auszuüben. Nach derzeitiger verfassungsrechtlicher Lage ist ein solcher Einsatz aber unzulässig.

\section{c) Weitere Ermächtigungsgrundlage zum Einsatz der Streitkräfte}

Eine Ermächtigungsgrundlage für den Einsatz der Streitkräfte zur Verteidigung im Innern sieht das Grundgesetz nicht vor. Die Streitkräfte dürfen gemäß Art. 87a Abs. 2 2. HS GG nur eingesetzt werden, soweit es das Grundgesetz ausdrücklich zulässt. Das Grundgesetz enthält hierzu in den Artt. 35, Art. 87a GG unterschiedliche Anspruchsvoraussetzungen. Die Normen lassen sich grob in zwei Kategorien einteilen, nämlich in rein technische Hilfeleistungen ohne Einsatzcharakter und in Einsätze im Innern im Sinne der o.g. Definition. Art und Umfang des Einsatzes hängen jeweils davon ab, welche Mittel die einschlägigen Normen freigeben. Diese Normen werden im Einzelnen untersucht:

\section{aa) Art. 35 Abs. 1 GG}

Unterstützungsmaßnahmen im Rahmen des Art. 35 Abs. 1 GG unterfallen nicht dem Verfassungsvorbehalt des Art. 87a Abs. 2 GG, da es sich um rein technische Maßnahmen unterhalb der Einsatzschwelle handelt. Hierzu zählen die technische Unterstützung der Polizei bei der Suche nach Vermissten oder die Bereitstellung von Soldaten als Arbeitskräfte bei Naturkatastrophen. ${ }^{28}$ Durch diese Form der Amtshilfe bleiben bestehende Zuständigkeiten und die Aufgabentrennung von Polizei und Streitkräften gewahrt. Im Rahmen des Art. 35 Abs. 1 GG können die Streitkräfte zur Terrorismusbekämpfung nur insoweit eingesetzt werden, als dass sie z.B. bei der Kampfmittelbeseitigung Amtshilfe leisten. ${ }^{29}$

\section{bb) Art. 35 Abs. 2 Satz 2 GG}

Ein Tätigwerden der Streitkräfte nach Art. 35 Abs. 2 Satz 2 GG kommt hauptsächlich im Rahmen der Katastrophenhilfe in Betracht und unterscheidet sich von der Amtshilfe des Abs. 1. Die Streitkräfte nehmen polizeiliche Aufgaben des anfordernden Bundeslandes wahr und handeln nach den spezifischen Landespolizeigesetzen. ${ }^{30}$ Der Einsatz typischer militärischer Gewalt ist daher von Art. 35 Abs. 2 Satz 2 GG nicht mehr erfasst. Selbst wenn man einen schweren Terrorangriff als Unglücksfall ansieht, dürfte die Verwendung militärischer Gewalt daran scheitern, dass es - abgesehen vom $\mathrm{UZwGBw}^{31}$ - keine gesetzliche Grundlage für ein Tätigwerden der Streitkräfte gibt. Mangels einer hierfür erforderlichen Ermächtigungsgrundlage können keine Zwangsmaßnahmen gegen Personen oder Sachen getroffen werden. ${ }^{32}$ Ein präventiver Einsatz der Streitkräfte (Aufklärung) scheitert bereits am

\footnotetext{
28 Weitere Beispiele bei Soria, DVBl. 2004, 597 (600).

29 Schmitdt-Jortzig, DÖV 2002, 773 (775).

30 Maunz, Maunz/Dürig, Grundgesetz-Kommentar, Art. 35 Rn. 20.

31 Gesetz über die Anwendung unmittelbaren Zwanges und die Ausübung besonderer Befugnisse durch die Soldaten der Bundeswehr und verbündete Streitkräfte sowie zivile Wachpersonen.

32 Sattler, 2004, 1286 (1289).
} 
Fehlen eines Unglückfalls. ${ }^{33}$ Für einen präventiven Einsatz im Bereich der Gefahrenabwehr spricht zwar, dass es hierfür ein höherrangiges Interesse gibt, und dies lasse ein Zuwarten bis zum Schadenseintritt nicht zu. Eine Auslegung der Verfassung unter reinen Effektivitätsgesichtspunkten ist aber rechtsdogmatisch fragwürdig und lässt sich mit dem Wortlaut der Norm nur mühevoll vereinbaren. Anders liegt der Fall, wenn sicher ist, dass ein Unglücksfall eintreten wird und es eine zeitlichräumliche Nähekomponente zwischen dem Einsatz der Streitkräfte und dem Unglücksfall gibt. Die Abwehr einer gegenwärtigen Gefahr - so die Formulierung im Polizeirecht - ist vom Sinn und Zweck des Art. 35 Abs. 2 Satz 2 GG gedeckt.

\section{cc) Art. 35 Abs. 3 GG}

Über Art. 35 Abs. 3 GG wird die Bundesregierung befugt, die Streitkräfte bei einer Gefährdungslage durch einen Unglücksfalls für mehr als ein Bundesland einzusetzen. Im Unterschied zu Abs. 2 ist eine Anforderung durch die betroffenen Länder nicht erforderlich. Umstritten ist, ob die Streitkräfte spezifisch-militärische Mittel im Rahmen des Art. 35 Abs. 3 GG einsetzen dürfen. Wegen der Bundeskompetenz zum Einsatz der Streitkräfte nach Abs. 3 wird vertreten, dass Landespolizeirecht keine Anwendung finde und der Bund alle ihm zur Verfügung stehenden Mittel, einschließlich militärischer Gewalt, einsetzen dürfe. ${ }^{34}$ Richtigerweise ändert das Kompetenzrecht des Bundes nichts an dem Arsenal der Waffen, das die Streitkräfte bei einem Einsatz im Innern einsetzen dürfen. Systematisch schließt Abs. 3 an die vorangegangenen Absätze an, wonach ein Einsatz der Streitkräfte mit Polizeimitteln zu erfolgen hat. Art. 35 Abs. 3 GG erweitert nicht die Wahl der Mittel, sondern nur die geographische Dimension des Einsatzes. ${ }^{35}$ Wie oben dargelegt könnten eben diese polizeilichen Mittel unzureichend sein, um bestimmten Bedrohungslagen wirksam begegnen zu können. Chemischen, radiologischen oder biologischen Bedrohungen, die etwa bei einem Angriff auf einen Chemietanker entstehen können, das gesamte Spektrum der Seeminenabwehr und die Abwehr von Unterwasserfahrzeugen, von See gestarteten Flugkörpern etc. sind mit polizeilichen Mitteln nicht darstellbar.

\section{dd) Art. $87 a$ Abs. 3 GG}

Nach Art. 87a Abs. 3 Satz 1 GG ist den Streitkräften unter den Voraussetzungen eines Spannungs- und Verteidigungsfalles das Recht eingeräumt, typische Polizeiaufgaben wahrzunehmen. Der Einsatz militärischer Mittel ist zulässig, da sie als »echtes « Militär tätig werden und nicht Teil einer Landespolizei sind, wie es gemäß Art. 35 Abs. 2, 3 GG der Fall ist. ${ }^{36}$ Der Auftrag der Streitkräfte ist begrenzt auf »den Schutz ziviler Objekte und Verkehrsregelung «, sie nehmen also Polizeifunktionen wahr. Unklar bleibt, unter welchen Einsatzregeln (rules of engagement) der Einsatz zu führen ist, ob originäres Polizeirecht mit seinem Verhältnismäßigkeitsgrundsatz oder ob Kriegsvölkerrecht Anwendung findet. Unabhängig von der

33 A.A: Baldus, NVwZ 2004, 1278 (1283).

$34 \mathrm{Vgl}$. Seifert/Bünker, ThürVBl. 200649 (55).

35 Soria, DVBl. 2004, 597 (603).

36 Seifert/Bünker, ThürVB1. 2006, 49 (55).
Beantwortung der Frage scheitert Art. 87a Abs. 3 Satz 1 GG als Ermächtigungsgrundlage für den Einsatz der Streitkräfte im Innern daran, dass er einen Verteidigungs- bzw. Spannungsfall voraussetzt. Die gegenwärtige abstrakte Bedrohung durch den Terrorismus reicht für einen solchen Fall auch unter Zugrundelegung eines erweiterten Verteidigungsbegriffs nicht aus. ${ }^{37}$ Ob ein konkreter Terroranschlag selbst hierzu ausreicht, hängt wohl von Art und Ausmaß des Anschlags ab. Eine bessere Verzahnung »äußerer und innerer Sicherheit « lässt sich auf Grundlage von Art. 87a Abs. 3 Satz 1 GG nicht erreichen.

\section{ee) Art. $87 a$ Abs. 3 Satz 2 GG}

Die Einsatzvoraussetzungen sind die gleichen wie bei Art. 87a Abs. 3 Satz 1 GG. Es bedarf der Feststellung eines Verteidigungs- bzw. Spannungsfalls. Der Umfang ist bis auf das Recht zur Verkehrsüberwachung ebenfalls mit Abs. 2 identisch. Als Ermächtigungsgrundlage für den Einsatz der Streitkräfte gegen terroristische Bedrohungen scheidet er daher aus.

\section{ff) Art. 87a Abs. 4 Satz 1 GG}

Das Grundgesetz ermächtigt gemäß Art. 87a Abs. 4 GG den Streitkräfteeinsatz bei einem inneren Notstand. Allgemein anerkannt ist, dass die Streitkräfte hierbei militärische Mittel einsetzen dürfen. ${ }^{38}$ Ein Einsatz der Streitkräfte zur Bekämpfung terroristischer Bedrohungen scheitert wiederum an den Einsatzvoraussetzungen der Norm. Diese sehen vor, dass der »Bestand des Bundes oder eines Landes in Gefahr ist « und die Polizeikräfte zur Gefahrenabwehr nicht ausreichen. Gemeint sind Situationen wie Bürgerkrieg oder Putsch, nicht hingegen Bedrohungen für sonstige Schutzgüter. ${ }^{39}$ Die Bedrohung der inneren Sicherheit durch Terrorakte kann nicht - jedenfalls nicht gegenwärtig - als eine Bedrohung für die verfassungsmäßige Ordnung angesehen werden.

\section{gg) Übergesetzlicher Notstand}

In Zusammenhang mit der Diskussion um den Abschuss einer von Terroristen gekaperten Passagiermaschine wurde bisweilen erwogen, ihren Abschuss mit militärischen Mittel als übergesetzlichen Notstand zu billigen. Sofern sich in der Maschine unschuldige Personen befinden, scheitert ein Abschuss am Abwägungsverbot des Art. 1 GG, wonach eine Abwägung »Leben gegen Leben « unzulässig ist. Aber selbst der Abschuss einer nur mit Terroristen besetzen Maschinen über unbewohntem Gebiet durch die Streitkräfte steht im Widerspruch zum ausdrücklichen Verfassungsvorbehalt des Art. 87a Abs. 2 GG. Zwar gelten diese Überlegungen auch für den Einsatz der Marine, doch stellt sich dieses spezielle Problem auf See so nicht. Denn auf See ist der Einsatz von militärischen Gewaltmitteln (Boarding, Zerstörung von Ruder/Antriebsanlage) möglich, die keine billigende Inkaufnahme der Tötung Unschuldiger erfordern.

\footnotetext{
37 In der Literatur wird bisweilen vertreten, »Verteidigung « umfasse auch terroristische Bedrohungen. Dem kann nicht gefolgt werden. m.w.N. Soria, DVBl. 2004, 597 (605)

38 Seifert/Bünker, ThürVBl. 2006, 49 (56).

39 Soria, DVBl. 2004, 597 (604)
} 


\section{Ergebnis}

Bereits nach geltender Rechtslage können die Streitkräfte zur Krisenbewältigung im Innern bei unterschiedlichen Szenarien eingesetzt werden, da diese sich größtenteils unterhalb der Einsatzschwelle befinden. Das gilt aber nicht für die zugespitzte Sicherheitslage durch terroristische Bedrohungen, wie die vorstehende verfassungsrechtliche Untersuchung gezeigt hat. ${ }^{40}$ Nachdem das Bundesverfassungsgericht mit Urteil vom 15.02.2006 entschied, dass § 14 Abs. 3 Luftsicherheitsgesetz nichtig ist, klafft eine Lücke zwischen realer Bedrohung und rechtlich zulässigen Abwehrmaßnahmen. Um der Politik klare Handlungsmöglichkeiten zu geben und das Handeln der Soldaten auf eine fundierte Rechtsgrundlage zu stellen, wird folgende Grundgesetzänderung vorgeschlagen:

\subsection{Vorschlag einer Grundgesetzänderung und Ergänzung}

Die vorgeschlagene Grundgesetzänderung bzw. Ergänzung berücksichtigt die erforderlichen Sicherheitsinteressen des Staates zur Abwehr erheblicher Gefahren im Innern, wenn die Kräfte der Polizei der Unterstützung durch die Streitkräfte bedürfen. Die Vorgaben des Bundesverfassungsgerichts aus dem Urteil zum Luftsicherheitsgesetz sind hierbei berücksichtigt worden.

\section{Neufassung des Art. 35 Abs. 4 GG}

Reichen zur Abwehr eines besonders schweren Unglücksfalls polizeiliche Mittel nicht aus, so kann die Bundesregierung den Einsatz der Streitkräfte mit militärischen Mitteln anordnen. Soweit es dabei zur wirksamen Bekämpfung erforderlich ist, kann die Bundesregierung den Landesregierungen Weisungen erteilen. Die Anordnung nach Satz 1 ist jederzeit auf Verlangen des Bundesrates, im Übrigen unverzüglich nach Beseitigung der Gefahr aufzuheben.

\section{Neufassung des Art 87a V GG}

Außerhalb des Hoheitsgebietes der Bundesrepublik Deutschland dürfen die Streitkräfte nach den Regeln des Völkerrechts, auch zur Unterstützung der zuständigen Bundesbehörden eingesetzt werden. Einsätze bedürfen der Zustimmung des Bundestages; das Nähere regelt ein Bundesgesetz.

\section{Begründung der Grundrechtsergänzung}

\section{Gründe für Art. 35 Abs. 4 GG (neu)}

Aus Sicht der Marine trägt die vorgeschlagene Ergänzung des Grundgesetzes mit dem Art. 35 Abs. 4 GG (neu) der angepassten Sicherheitslage vollumfänglich Rechnung. Das enorme Gefahrenpotential des internationalen Terrorismus steht

40 So auch Seifert/Bünker, ThürVBl. 2006, 49 (57). nicht mehr im Einklang mit der Notstandsverfassung, bei deren Erschaffung die Verteilung polizeilicher und militärischer Aufgaben die heutige Situation nicht berücksichtigen konnte. Der Zugang zu modernen Technologien und die Privatisierung ursprünglich staatlicher Gewaltmittel haben potentiellen Terroristen einen Vorsprung vor den Polizeikräften verschafft. Der vorgeschlagene Artikel ermöglicht die Unterstützung der Vollzugsbehörden durch die Streitkräfte unter Einbeziehung militärischer Mittel etwa im Rahmen der Absicherung des G-8 Gipfels, des Bush-Besuchs oder ähnlicher Szenarien, bei denen dies nach geltendem Recht nicht zulässig ist. Die parlamentarische Kontrolle bleibt bei diesem Vorschlag erhalten. Durch die Regelungen des Art. 35 III 1 GG und die Formulierungen des Art. 35 III GG wird weiterhin sichergestellt, dass die originären Zuständigkeiten der bislang für die Gefahrenabwehr zuständigen Behörden nicht berührt werden. Nur dann, wenn deren Kräfte nicht ausreichen - »im besonders schweren Unglücksfall «-, könnte die Marine mit spezifisch militärischen Mitteln tätig werden.

\section{Gründe für Art. 87a V (neu)}

Die Bundesrepublik Deutschland hat sich in zahlreichen internationalen Verträgen und Initiativen verpflichtet, Maßnahmen gegen bestimmte Bedrohungen zu ergreifen. Dazu zählen u.a. die Bekämpfung der Piraterie, die im 3. Seerechtsübereinkommen von 1982 festgeschrieben wurde, sowie Maßnahmen gegen die Verbreitung von Massenvernichtungswaffen, die im Rahmen der PSI-Initiative vorgesehen sind. Nach geltendem Recht besteht, wie oben dargelegt, eine erhebliche Diskrepanz zwischen völkerrechtlichen Verpflichtungen und den dafür bereitgestellten Mitteln. Die Marine erfüllt diese und ähnliche Aufgaben bereits seit Jahren im Kontext internationaler Einsätze, die durch den Bundestag mandatiert sind und ihre Grundlage in Art. 24 II GG haben. So überwachen Schiffe der Marine beispielsweise den Seeraum am Horn von Afrika und dürfen bei aufgedeckten Aktivitäten des internationalen Terrorismus tätig werden. Entdecken Marineeinheiten dort oder anderswo hingegen noch so brutale Aktivitäten der Piraterie, fehlt ihnen nach nationalem Recht jede Eingriffsbefugnis. Diese Lücke wird durch Art. 87 a V (neu) geschlossen. Auch hier bleiben die originären Befugnisse der übrigen Behörden unangetastet. Subsidiär und unter voller parlamentarischer Kontrolle könnten der Marine nach Maßgabe dieses Artikels aber Befugnisse eingeräumt werden, die die völkerrechtlichen Verpflichtungen mit nationalem Recht in Deckung bringen.

Der Staat und die Streitkräfte sind dem Schutz des Grundgesetzes verpflichtet. Dazu zählen auch völkerrechtlich verbindlich eingegangene Verpflichtungen durch die Ratifikation entsprechender Verträge. Ein Hinweis auf unzulängliche nationale Zuständigkeitsregelungen wird der Verantwortung Deutschlands nicht gerecht und bedarf daher entsprechender Anpassung. Diese wird durch den vorgeschlagenen Art. 87a V (neu) gewährleistet. 


\section{Zusammenfassung}

Das Thema »Maritime Sicherheit « wird trotz der herausragenden Bedeutung, die der maritime Raum für Deutschland aus wirtschaftlichen und sicherheitspolitischen Gründen hat, in der Debatte um die innere Sicherheit nur sporadisch reflektiert. Demgegenüber zielen zahlreiche internationale Initiativen darauf $\mathrm{ab}$, das Thema ins öffentliche Bewusstsein zu rücken und Fortschritte zu erreichen. Im internationalen Kontext muss es darum gehen, diese Initiativen $\mathrm{zu}$ harmonisieren und zu bündeln und die Voraussetzungen für ein umfassendes maritimes Lagebild und einen entsprechenden Informationsaustausch zu schaffen. Allen Bemühungen liegt die Erkenntnis zugrunde, dass sich Erfolge auf dem Gebiet nur in einem ganzheitlichen, ressortübergreifenden und internationalen Ansatz erzielen lassen.

In Deutschland sind zwar mit der Aufstellung des Organisationsverbundes Küstenwache und der Inbetriebnahme des Gemeinsamen Lagezentrums im Maritimen Sicherheitszentrum beachtenswerte Schritte in die richtige Richtung erfolgt, es bedarf aber einer umfassenden und rechtlich abgesicherten Einbindung der Marine als dem maritimen Kompetenzträger. Diese Einbindung verlangt verfassungsrechtliche Änderungen als Grundlage dringend notwendiger Handlungskompetenzen.

\section{Transformation, Development, and Regionalization in Greater Asia}

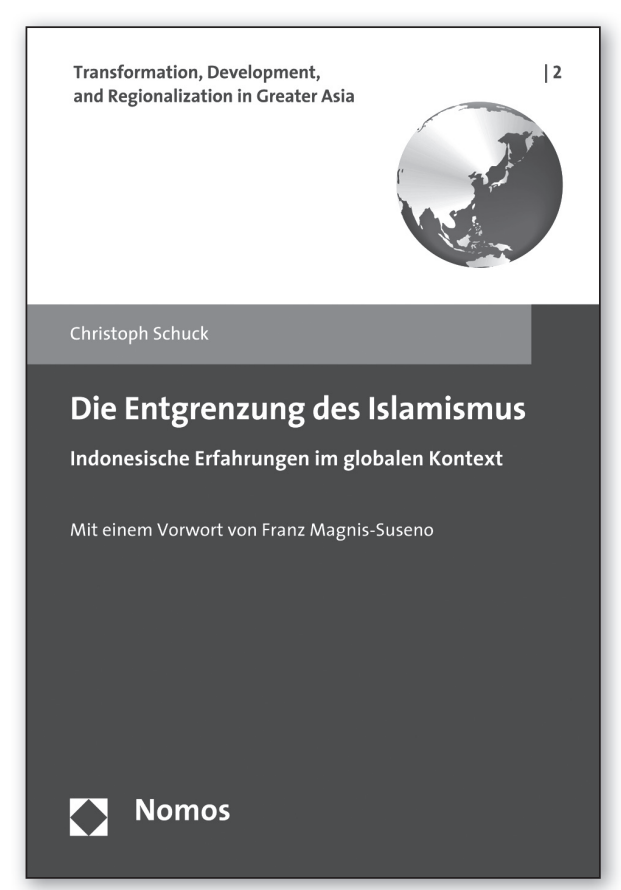

\author{
Die Entgrenzung des Islamismus \\ Indonesische Erfahrungen im globalen Kontext \\ Von Christoph Schuck \\ Mit einem Vorwort von Franz Magnis-Suseno \\ 2008, Band 2, 265 S., brosch., 39,- - ISBN 978-3-8329-3191-9
}

Dieses Buch setzt sich mit dem Phänomen der Intensivierung und Entgrenzung des Islamismus in einer zunehmend „borderless world“ sowohl in allgemeiner als auch in speziell auf Indonesien gerichteter Form auseinander und verbindet diese beiden Blickwinkel. Dabei wird eine Modellierung des Islamismus vorgenommen, die Entgrenzungstendenz herausgearbeitet und diese an konkreten Fallbeispielen analysiert. 